\title{
On equations defining fake elliptic curves
}

\author{
par PILAR BAYER et JoRdi GUÀRDIA
}

\begin{abstract}
RÉSUMÉ. Les courbes de Shimura associées à des algèbres de quaternions rationnelles et non décomposées forment des espaces de modules grossiers pour les surfaces abeliennes principalement polarisées munies d'une multiplication par les quaternions. Ces objets sont également connus sous le nom de fausses courbes elliptiques. Nous présentons une méthode pour calculer des équations de courbes de genre 2 dont la Jacobienne est une fausse courbe elliptique avec multiplication complexe. La méthode est basée sur la connaissance explicite des matrices de périodes normalisées et sur l'utilisation de fonctions theta avec caractéristiques. Comme dans le cas des points CM sur les courbes modulaires classiques, les fausses courbes elliptiques CM jouent un role clé dans la construction des corps de classes au moyen des valeurs spéciales des fonctions automorphes (cf. [Sh67]).
\end{abstract}

ABSTRACT. Shimura curves associated to rational nonsplit quaternion algebras are coarse moduli spaces for principally polarized abelian surfaces endowed with quaternionic multiplication. These objects are also known as fake elliptic curves. We present a method for computing equations for genus 2 curves whose Jacobian is a fake elliptic curve with complex multiplication. The method is based on the explicit knowledge of the normalized period matrices and on the use of theta functions with characteristics. As in the case of CM-points on classical modular curves, CMfake elliptic curves play a key role in the construction of class fields by means of special values of automorphic functions (cf. [Sh67]).

\section{Contents}

1. Fake elliptic curves and their moduli 58

2. Genus 2 curves and theta functions 60

3. Algebraic models for genus 2 curves $\quad 62$

$\begin{array}{ll}\text { 4. The equations } & 63\end{array}$

$\begin{array}{ll}\text { References } & 67\end{array}$

Partially supported by MCYT BFM2000-0627, BFM2003-01898 and BFM2003-06768-C02-02. 


\section{Fake elliptic curves and their moduli}

We consider a rational nonsplit quaternion algebra $H=\left(\frac{a, b}{\mathbb{Q}}\right)$ with basis $\{1, \mathrm{I}, \mathrm{J}, \mathrm{IJ}\}$ satisfying the following relations

$$
\mathrm{I}^{2}=a, \quad \mathrm{~J}^{2}=b, \quad \mathrm{IJ}=-\mathrm{JI} \quad\left(a, b \in \mathbb{Q}^{*}\right) .
$$

We suppose that $H$ splits at infinity, that is $H \otimes_{\mathbb{Q}} \mathbb{R} \simeq M(2, \mathbb{R})$, and call $D=p_{1} \ldots p_{2 r}$ the discriminant of $H$, which is equal to the product of an even number of prime numbers. We denote by

$$
h: \mathbb{C}^{*} \rightarrow H^{*}(\mathbb{R}) \simeq \mathrm{GL}(2, \mathbb{R})
$$

the group homomorphism such that $h(i)$ is the right multiplication by $\left[\begin{array}{cc}0 & 1 \\ -1 & 0\end{array}\right]$

The main anti-involution of $H$

$$
\alpha=x+y \mathrm{I}+z \mathrm{~J}+t \mathrm{IJ} \mapsto \bar{\alpha}=x-y \mathrm{I}-z \mathrm{~J}-t \mathrm{IJ} \quad(\alpha \in H)
$$

gives rise to the reduced trace and reduced norm of $H$

$$
\operatorname{Tr}(\alpha)=\alpha+\bar{\alpha}, \quad \mathrm{N}(\alpha)=\alpha \bar{\alpha} \quad(\alpha \in H) .
$$

According to Shimura (cf. [Sh67]), a PEL-type

$$
\Omega=(H, \Phi, * ; \mathrm{T}, \mathcal{O} ; \mathrm{V})
$$

consists of an embedding of $\mathbb{Q}$-algebras $\Phi: H \hookrightarrow M(2, \mathbb{R})$, a positive antiinvolution $*$ of $H$; a maximal order $\mathcal{O} \subseteq H$, a nondegenerate alternating form $\mathrm{T}$ on $H$, integer valued over $\mathcal{O}$; and a level structure $\mathrm{V}=\left(v_{1}, \ldots, v_{s}\right)$ provided by finitely many ordered quaternion classes $v_{i} \in \mathcal{O} \otimes \mathbb{Q} / \mathcal{O}=H / \mathcal{O}$.

From the Skolem-Noether theorem, it follows that there exists a quaternion $\mu \in H$ such that $\alpha^{*}=\mu^{-1} \bar{\alpha} \mu$ for any $\alpha \in H$. The element $\mu$ turns out to be a pure quaternion $(\bar{\mu}=-\mu)$ for which $\mu^{2}<0$, because $*$ is positive. Since the field $\mathbb{Q}(\sqrt{-D})$ splits $H, \mu$ may be chosen so that $\mu \in \mathcal{O}$ and $\mu^{2}=-D$. The lattice $\mathcal{O}$ then becomes stable under $*$. We shall fix a PEL-type throughout with the element $\mu$ chosen in this way.

Definition. A fake elliptic curve $(A, \iota, \mathcal{L}, W)$ over $\mathbb{C}$ of PEL-type $\Omega$ consists of

(i) An abelian surface $A / \mathbb{C}$.

(ii) An injective ring homomorphism $\iota: \mathcal{O} \hookrightarrow \operatorname{End}(A)$ such that $H_{1}(A, \mathbb{Z})$ regarded as a left $\mathcal{O}$-module is isomorphic to $\mathcal{O}$.

(iii) A principal polarization $\mathcal{L}$ on $A$ such that the associated Rosati antiinvolution, $\phi_{\mathcal{L}}: \operatorname{End}^{0}(A) \rightarrow \operatorname{End}^{0}(A)$, restricts to the anti-involution $*$ on $\iota(\mathcal{O})$.

(iv) A level structure $\mathrm{W} \subset H_{1}(A, \mathbb{Q})$ given by the image of $\mathrm{V}$ under the isomorphism $H \simeq H_{1}(A, \mathbb{Q})$ obtained from (ii). 
As usual, we have written $\operatorname{End}^{0}(A)$ for $\operatorname{End}(A) \otimes \mathbb{Q}$.

Let $\mathcal{H}$ denote the Poincaré upper half-plane. The group $\mathcal{O}_{1}^{*}$ of units of $\mathcal{O}$ of reduced norm equal one gives rise to a Fuchsian group

$$
\Gamma_{D}:=\Phi\left(\mathcal{O}_{1}^{*}\right) \subseteq \mathrm{SL}(2, \mathbb{R}) .
$$

Associated with the action of $\Gamma_{D}$ on $\mathcal{H}$ by fractional linear transformations, there is a complex projective non-singular curve which is a solution of a moduli problem. Shimura proved the existence of a canonical model, $\left(X_{D}, j_{D}\right)$, where the curve $X_{D}$ is defined over $\mathbb{Q}$ and the canonical mapping

$$
j_{D}: \Gamma_{D} \backslash \mathcal{H} \longrightarrow X_{D}(\mathbb{C})
$$

is one to one. Moreover, the complex points of $X_{D}$ are in one to one correspondence with the isomorphism classes $[A, \iota, \mathcal{L}]$ of fake elliptic curves of PEL-type $\Omega=(H, \Phi, * ; \mathrm{T}, \mathcal{O} ; 0)$.

Now, let us consider an imaginary quadratic field $F=\mathbb{Q}(\sqrt{d}), d<0$, and let $R=R(d, m) \subseteq F$ be a quadratic order of conductor $m$. Suppose that there is an embedding $\varphi: F \hookrightarrow H$ or, equivalently, that the field $F$ splits $H$. A fake elliptic curve $(A, \iota, \mathcal{L})$ is said to be of complex multiplication $(\mathrm{CM})$ by $R$ if the embedding $\varphi$ restricts to an optimal embedding $\varphi: R \hookrightarrow \mathcal{O}$ and

$$
\operatorname{End}((A, \iota, \mathcal{L})) \simeq R
$$

If this is the case,

$$
\operatorname{End}^{0}((A, \iota, \mathcal{L})) \simeq F, \quad \operatorname{End}^{0}(A) \simeq H \otimes_{\mathbb{Q}} F \simeq M(2, F),
$$

and the abelian variety $A$ is isogenous to the square of an elliptic curve with complex multiplication. Then the class $[A, \iota, \mathcal{L}]$ provides a CM-point on the Shimura curve $X_{D}$. CM-points on Shimura curves are relevant since their coordinates in the canonical model generate class fields. Indeed, the embedding $\varphi$ yields a point $\tau \in \mathcal{H}$, fixed under all transformations in $\Phi(\varphi(F))$, for which $(A, \iota, \mathcal{L})=\left(A_{\tau}, \iota_{\tau}, \mathcal{L}_{\tau}\right)$ and $j_{D} \circ \pi(\tau) \in X_{D}\left(F^{a b}\right)$. Here $\pi: \mathcal{H} \rightarrow \Gamma_{D} \backslash \mathcal{H}$ denotes the canonical projection. The behaviour of the CM-points under the Frobenius automorphisms is governed by Shimura's reciprocity law (cf. [Sh67]).

A CM-point $\tau \in \mathcal{H}$ will be called special (SCM) if it is the fixed point of some fractional linear transformation $\gamma \in \Phi \circ \varphi(R(d, m))$ of determinant equal to $D$. The binary normic form $n_{F}(X, Y)=X^{2}-d Y^{2}$ of the field $F=\mathbb{Q}(\sqrt{d})$ yields a binary quadratic form when restricted to $R(d, m)=$ $\mathbb{Z}[1, m \omega]$, where $\omega=\sqrt{d}$ if $d \equiv 2,3(\bmod 4)$, and $\omega=\frac{1+\sqrt{d}}{2}$ if $d \equiv 1(\bmod 4)$. 
This form is given by

$$
n_{R}(X, Y)= \begin{cases}X^{2}-\frac{D_{F}}{4} m^{2} Y^{2}, & \text { if } D_{F} \equiv 0(\bmod 4), \\ X^{2}+m X Y+m^{2} \frac{1-D_{F}}{4} Y^{2}, & \text { if } D_{F} \equiv 1(\bmod 4) .\end{cases}
$$

The SCM-points are obtained from quadratic orders $R$ for which $D$ has an integral representation by $n_{R}(X, Y)$. Moreover, their orbits under $\Gamma_{D}$ are finite in number (cf. [Al04], [AlBa04]).

\section{Genus 2 curves and theta functions}

Let $\mathcal{H}_{2}$ denote the Siegel upper half-space whose elements are the $2 \times 2$ symmetric matrices with positive definite imaginary part

$$
\mathcal{H}_{2}=\left\{Z \in M(2, \mathbb{C}): Z={ }^{t} Z, \operatorname{Im} Z>0\right\} .
$$

We consider the Riemann theta function

$$
\vartheta(z ; Z)=\sum_{n \in \mathbb{Z}^{2}} \exp \left(\pi i^{t} n \cdot Z \cdot n+2 \pi i^{t} n \cdot z\right) \quad\left(z \in \mathbb{C}^{2}, Z \in \mathcal{H}_{2}\right) .
$$

Given a pair $(r, s)$ of column vectors, $r, s \in\{0,1 / 2\}^{2}$, let $c={ }^{t}[r, s]$. The theta function with half-integral characteristic $c$ is defined by

$$
\vartheta[c](z ; Z)=\exp \left(\pi i^{t} r \cdot Z \cdot r+2 \pi i^{t} r \cdot(z+s)\right) \vartheta(z+Z \cdot r+s ; Z) .
$$

It is an even or odd function of $z$ according to the parity of $c$ :

$$
\vartheta[c](z ; Z) \text { is }\left\{\begin{array} { l } 
{ \text { even } } \\
{ \text { odd } }
\end{array} 4 ^ { t } r \cdot s \equiv \left\{\begin{array}{ll}
0 & (\bmod 2) .
\end{array}\right.\right.
$$

For a fixed matrix $Z \in \mathcal{H}_{2}$, half-integral characteristics are in bijection with 2 -torsion points in the complex torus $J_{Z}:=\mathbb{C}^{2} /\left\langle 1_{2}, Z\right\rangle$ by means of the correspondence

$$
c={ }^{t}[r, s] \longleftrightarrow w=Z \cdot r+s \in J_{Z}[2] .
$$

We shall use the symbols $c, c_{k}, \ldots$ for half-integral characteristics, and the symbols $w, w_{k}, \ldots$ for the corresponding 2-torsion points on $J_{Z}$. Accordingly, we define the parity of a 2-torsion point as the parity of its characteristic.

Let $C$ be the smooth projective curve with hyperelliptic model

$$
Y^{2}=f(X)
$$

where $f(X)=a_{6} X^{6}+\cdots+a_{1} X+a_{0} \in \mathbb{C}[X]$ is a polynomial of degree $n=5$ or 6 without multiple roots. It is of genus $g=\lfloor(n-1) / 2\rfloor=2$. We have that

$$
H^{0}\left(C, \Omega_{C}^{1}\right)=\left\langle\omega_{1}=\frac{d x}{y}, \omega_{2}=\frac{x d x}{y}\right\rangle, \quad H_{1}(C, \mathbb{Z})=\left\langle\gamma_{1}, \gamma_{2}, \gamma_{3}, \gamma_{4}\right\rangle
$$


where $\Omega_{C}^{1}$ stands for the sheaf of holomorphic differentials on $C$, and the basis of $H_{1}(C, \mathbb{Z})$ is symplectic for the intersection pairing. The period matrix attached to these data

$$
\Omega_{f}=\left[\begin{array}{cccc}
\int_{\gamma_{1}} \omega_{1} & \int_{\gamma_{2}} \omega_{1} & \int_{\gamma_{3}} \omega_{1} & \int_{\gamma_{4}} \omega_{1} \\
\int_{\gamma_{1}} \omega_{2} & \int_{\gamma_{2}} \omega_{2} & \int_{\gamma_{3}} \omega_{2} & \int_{\gamma_{4}} \omega_{2}
\end{array}\right]=\left[\Omega_{1, f} \mid \Omega_{2, f}\right]
$$

defines a point $Z_{f}:=\Omega_{1, f}^{-1} \Omega_{2, f}$ in $\mathcal{H}_{2}$. By setting

$$
\left[\begin{array}{l}
\eta_{1} \\
\eta_{2}
\end{array}\right]=\Omega_{1, f}^{-1}\left[\begin{array}{l}
\omega_{1} \\
\omega_{2}
\end{array}\right]
$$

we obtain a second basis $\left\{\eta_{1}, \eta_{2}\right\}$ of $H^{0}\left(C, \Omega_{C}^{1}\right)$. The period matrix of $C$ with respect to this new basis is $\left[1_{2} \mid Z_{f}\right]$. We will think of the the Jacobian variety of $C$ as the complex torus $J(C)=\mathbb{C}^{2} /\left\langle 1_{2}, Z_{f}\right\rangle$.

We fix a Weierstrass point $W \in C$ as the base point for the Abel-Jacobi mapping defined by

$$
\begin{aligned}
\Pi: C & \rightarrow J(C) \\
P & \rightarrow \int_{W}^{P}\left(\eta_{1}, \eta_{2}\right)+\kappa,
\end{aligned}
$$

where $\kappa$ is the Riemann vector. It guarantees that

$$
\Pi(C)=\Theta-\kappa,
$$

being $\Theta$ the divisor of the Riemann theta function $\vartheta(z ; Z)$.

Let $\alpha_{k}$ denote the roots of $f$. By means of $\Pi$, the six Weierstrass points $W_{k}=\left(\alpha_{k}, 0\right)$ of $C$ give rise to six odd 2-torsion points in its Jacobian:

$$
w_{k}=\Pi\left(W_{k}\right) \in J(C)[2]^{\text {odd }} .
$$

If we let

$$
m_{1}=\left[\begin{array}{l}
0 \\
0
\end{array}\right], m_{2}=\left[\begin{array}{c}
0 \\
1 / 2
\end{array}\right], m_{3}=\left[\begin{array}{c}
1 / 2 \\
0
\end{array}\right], m_{4}=\left[\begin{array}{l}
1 / 2 \\
1 / 2
\end{array}\right],
$$

then the points $w_{k}$ are represented by $z_{j, k}:=m_{j}+Z_{f} \cdot m_{k}$, for

$$
(j, k) \in\{(2,2),(4,2),(3,3),(4,3),(2,4),(3,4)\} .
$$

The following result, which we formulate for genus 2 curves, is a particular case of a theorem proved in Guàrdia [Gu02], valid for any genus.

Theorem 2.1. Suppose that $f(X)=a_{6} X^{6}+a_{5} X^{5}+\cdots+a_{0} \in \mathbb{C}[X]$ is a polynomial of degree 5 or 6 without multiple roots. Let $C: Y^{2}=f(X)$. Then the roots of $f(X)$ are the ratios $\alpha_{k}=x_{k, 2} / x_{k, 1}$, obtained through the solutions $\left(x_{k, 1}, x_{k, 2}\right)$ of the linear homogeneous equation

$$
H_{k}\left(X_{1}, X_{2}\right):=\left[\frac{\partial \vartheta}{\partial z_{1}}\left(w_{k}, Z_{f}\right) \frac{\partial \vartheta}{\partial z_{2}}\left(w_{k}, Z_{f}\right)\right] \Omega_{1, f}^{-1}\left[\begin{array}{c}
X_{1} \\
X_{2}
\end{array}\right]=0
$$


for $w_{k} \in J\left(C_{f}\right)[2]^{\text {odd }}, 1 \leq k \leq 6$. Here $\Omega_{f}=\left[\Omega_{1, f} \mid \Omega_{2, f}\right]$ is the period matrix of $C$ with respect to the basis $\left\{\frac{d x}{y}, x \frac{d x}{y}\right\}$ of $H^{0}\left(C, \Omega_{C}^{1}\right)$, and to any symplectic basis of $H_{1}(C, \mathbb{Z}) ; Z_{f}=\Omega_{1, f}^{-1} \Omega_{2, f}$.

\section{Algebraic models for genus 2 curves}

Now suppose that we are given a point $Z \in \mathcal{H}_{2}$ and consider the torus $J_{Z}$ attached to it. Given a pair $\left(c_{1}, c_{2}\right)$ of different odd characteristics and the corresponding pair $\left(w_{1}, w_{2}\right)$ of odd 2-torsion points in $J_{Z}$, we define

$$
J\left(c_{1}, c_{2}\right)=J\left(w_{1}, w_{2}\right)=\left[\begin{array}{ll}
\frac{\partial \vartheta\left[c_{1}\right]}{\partial z_{1}}(0 ; Z) & \frac{\partial \vartheta\left[c_{1}\right]}{\partial z_{2}}(0 ; Z) \\
\frac{\partial \vartheta\left[c_{2}\right]}{\partial z_{1}}(0 ; Z) & \frac{\partial \vartheta\left[c_{2}\right]}{\partial z_{2}}(0 ; Z)
\end{array}\right]
$$

and

$$
\left[c_{1}, c_{2}\right]=\left[w_{1}, w_{2}\right]=\operatorname{det} J\left(w_{1}, w_{2}\right) .
$$

If $\left[1_{2} \mid Z\right]$ is the normalized period matrix of a hyperelliptic curve $C / k$, the following theorem tells us how to compute an equation for this curve with coefficients in an algebraic closure $\bar{k}$.

Theorem 3.1. (Guàrdia [Gu]) Let $C / k$ be a genus 2 curve and let $Z \in \mathcal{H}_{2}$ define a normalized period matrix for $C$. We identify $J(C)$ with $J_{Z}$ and fix a pair of points $\left(w_{1}, w_{2}\right), w_{i} \in J(C)[2]^{\text {odd }}, w_{1} \neq w_{2}$.

(i) For any $w \in J(C)[2]^{\text {odd }}$, the point in $\mathbb{P}^{1}(\mathbb{C})$ cut by the hyperplane

$$
\left[\frac{\partial \vartheta}{\partial z_{1}}(w ; Z) \quad \frac{\partial \vartheta}{\partial z_{2}}(w ; Z)\right] J\left(w_{1}, w_{2}\right)^{-1}\left[\begin{array}{l}
X_{1} \\
X_{2}
\end{array}\right]=0
$$

has projective coordinates $\left(\left[w_{1}, w\right]:\left[w_{2}, w\right]\right)$.

(ii) Let $J(C)[2]^{\text {odd }}=\left\{w_{i}\right\}_{1 \leq i \leq 6}$. The ratios

$$
\ell_{12 j}:=\frac{\left[w_{2}, w_{j}\right]}{\left[w_{1}, w_{j}\right]}, \quad 1 \leq j \leq 6,
$$

are either $\infty$ (for $j=1$ ) or algebraic over $k$.

(iii) The curve $C$ admits the hyperelliptic model

$$
Y^{2}=X\left(X-\ell_{123}\right)\left(X-\ell_{124}\right)\left(X-\ell_{125}\right)\left(X-\ell_{126}\right),
$$

which is defined over a finite extension of $k$.

Note that theorem 3.1 yields thirty models for the isomorphism class of our curve, which correspond to the thirty possible choices of ordered pairs $\left(w_{1}, w_{2}\right)$. 


\section{The equations}

We now consider the Shimura curves $X_{D}$ for $D=6,10,15$, and all the SCM-points on them. By applying theorem 3.1, we obtain equations for genus 2 curves whose Jacobians are SCM-fake elliptic curves, defined by SCM-points $\tau \in \mathcal{H}$. These points will be calculated in accordance with [AlBa04].

The SCM-fake elliptic curve $\left(A_{\tau}, \iota_{\tau}, \mathcal{L}_{\tau}, W_{\tau}\right)$ will be obtained as follows:

$$
\Lambda_{\tau}=\Phi\left(\mathcal{O}_{D}\right)\left[\begin{array}{l}
\tau \\
1
\end{array}\right], \quad \mathbb{C}^{2} / \Lambda_{\tau} \simeq A_{\tau}(\mathbb{C}) .
$$

We define $\mathrm{T}(\alpha, \beta):=\operatorname{Tr}(\mu \alpha \bar{\beta})$, where $\mu \in D, \mu^{2}=-D$ and $\alpha, \beta \in H$. We choose in $\Phi\left(\mathcal{O}_{D}\right)$ a symplectic basis with respect to the bilinear form $E(\Phi(\alpha), \Phi(\beta)):=\delta^{-1} \mathrm{~T}(\alpha, \beta)$, where $\delta:=(\operatorname{Pf}(\mathrm{T}))^{1 / 2}$ denotes the positive square root of the Pfaffian of T. The principal polarization $\mathcal{L}_{\tau}$ will be given by the Riemann form

$$
\begin{array}{cccc}
E_{\tau}: & \Lambda_{\tau} \times \Lambda_{\tau} & \longrightarrow & \mathbb{C} \\
(\Phi(\alpha) \tau, \Phi(\beta) \tau) & \longrightarrow & E(\Phi(\alpha), \Phi(\beta)) .
\end{array}
$$

The level structure $\mathrm{V}=\left(v_{1}, v_{2}\right)$ will be associated with an ordered pair $\left(v_{1}, v_{2}\right), v_{i} \in H / \mathcal{O}$, such that $w_{i}:=\Phi\left(v_{i}\right) \tau \in \mathbb{C}^{2} / \Lambda_{\tau}[2]^{\text {odd }}$; then $W:=$ $\left(w_{1}, w_{2}\right)$. In each example, we have fixed one of the thirty possible level structures.

Example 1. $H=\left(\frac{3,-1}{\mathbb{Q}}\right)=\langle 1, \mathrm{I}, \mathrm{J}, \mathrm{IJ}\rangle, D=6$.

We consider the PEL-type given by the following data:

$\Phi(\mathrm{I})=\left[\begin{array}{cc}\sqrt{3} & 0 \\ 0 & -\sqrt{3}\end{array}\right], \Phi(\mathrm{J})=\left[\begin{array}{cc}0 & 1 \\ -1 & 0\end{array}\right]$,

$\mu=\mathrm{I}+3 \mathrm{~J}, \quad \delta=6$,

$\mathcal{O}_{6}=\mathbb{Z}\left[1, \mathrm{I}, \mathrm{J}, \frac{1+\mathrm{I}+\mathrm{J}+\mathrm{IJ}}{2}\right]$,

$\mathrm{V}=\left(\left(\frac{1}{4}, \frac{1}{4},-\frac{3}{4}, \frac{1}{4}\right),\left(\frac{1}{4}, \frac{1}{4},-\frac{1}{4}, \frac{1}{4}\right)\right)$,

$\Phi\left(\mathcal{O}_{6}\right)=\mathbb{Z}\left[1_{2},\left[\begin{array}{cc}\frac{-1+\sqrt{3}}{2} & \frac{-1+\sqrt{3}}{2} \\ \frac{1+\sqrt{3}}{2} & \frac{-1-\sqrt{3}}{2}\end{array}\right],\left[\begin{array}{cc}0 & 1 \\ -1 & 0\end{array}\right],\left[\begin{array}{cc}1-\sqrt{3} & 0 \\ 0 & 1+\sqrt{3}\end{array}\right]\right]$.

We have two SCM-points, which have CM by the maximal order $R(-6,1)$ :

$$
\tau_{1}=\frac{i(\sqrt{6}-\sqrt{2})}{2}, \quad \tau_{2}=\frac{\sqrt{3}+i \sqrt{6}}{3} .
$$


They define the points in the Siegel upper half-space

$$
Z_{1}=\left[\begin{array}{ll}
\frac{-1+3 i \sqrt{6}}{5} & \frac{-2+i \sqrt{6}}{5} \\
\frac{-2+i \sqrt{6}}{5} & \frac{6+2 i \sqrt{6}}{5}
\end{array}\right], Z_{2}=\left[\begin{array}{cc}
\frac{-1+2 i \sqrt{6}}{5} & \frac{-2-i \sqrt{6}}{5} \\
\frac{-2-i \sqrt{6}}{5} & \frac{6+3 i \sqrt{6}}{5}
\end{array}\right],
$$

and yield the genus 2 curves

$$
\begin{gathered}
Y^{2}=X^{5}-2 i \sqrt{2} X^{4}-\frac{11}{3} X^{3}+2 i \sqrt{2} X^{2}+X \\
Y^{2}=X^{5}+2 \sqrt[4]{-5+2 \sqrt{6}} X^{4}-\frac{10}{3} i \sqrt{2} X^{3}+2 i \sqrt[4]{-5-2 \sqrt{6}} X^{2}+X .
\end{gathered}
$$

Their common Igusa's invariants are

$$
\begin{array}{ll}
i_{1}=-\frac{322102}{3} & =-\frac{2 \cdot 11^{5}}{3}, \\
i_{2}=23958 & =2 \cdot 3^{2} \cdot 11^{3}, \\
i_{3}=5082 & =2 \cdot 3 \cdot 7 \cdot 11^{2} .
\end{array}
$$

Example 2. $H=\left(\frac{2,5}{\mathbb{Q}}\right)=\langle 1, \mathrm{I}, \mathrm{J}, \mathrm{IJ}\rangle, D=10$.

We consider the PEL-type given by the following data:

$\Phi(\mathrm{I})=\left[\begin{array}{cc}\sqrt{2} & 0 \\ 0 & -\sqrt{2}\end{array}\right], \Phi(\mathrm{J})=\left[\begin{array}{cc}0 & 1 \\ -1 & 0\end{array}\right]$,

$\mu=-\mathrm{IJ}, \quad \delta=10$,

$\mathcal{O}_{10}=\mathbb{Z}\left[1, \mathrm{I}, \frac{1+\mathrm{J}}{2}, \frac{\mathrm{I}+\mathrm{IJ}}{2}\right]$,

$\mathrm{V}=\left(\left(\frac{1}{4}, \frac{1}{2},-\frac{1}{4}, 0\right),\left(\frac{1}{2}, \frac{1}{2}, 0,0\right)\right)$,

$\Phi\left(\mathcal{O}_{10}\right)=\mathbb{Z}\left[1_{2},\left[\begin{array}{cc}\sqrt{2} & 0 \\ 0 & -\sqrt{2}\end{array}\right],\left[\begin{array}{cc}-\frac{\sqrt{2}}{2} & -\frac{\sqrt{2}}{2} \\ \frac{5 \sqrt{2}}{2} & \frac{\sqrt{2}}{2}\end{array}\right],\left[\begin{array}{cc}-\frac{1}{2} & -\frac{1}{2} \\ -\frac{5}{2} & -\frac{1}{2}\end{array}\right]\right.$.

We have two SCM-points, which have CM by the maximal order $R(-10,1)$ :

$$
\tau_{1}=\frac{i(3 \sqrt{5}-2 \sqrt{10})}{5}, \quad \tau_{2}=\frac{i \sqrt{5}}{5} .
$$

They define the points in the Siegel upper half-space

$$
Z_{1}=\left[\begin{array}{cc}
\frac{3 i \sqrt{10}}{2} & -\frac{1}{2}+i \sqrt{10} \\
-\frac{1}{2}+i \sqrt{10} & \frac{3 i \sqrt{10}}{4}
\end{array}\right], \quad Z_{2}=\left[\begin{array}{cc}
\frac{i \sqrt{10}}{2} & -\frac{1}{2} \\
-\frac{1}{2} & \frac{i \sqrt{10}}{4}
\end{array}\right] .
$$

and yield the genus 2 curves

$$
\begin{aligned}
& Y^{2}=X^{5}+2 \sqrt{5} X^{4}+\frac{125}{18} X^{3}+2 \sqrt{5} X^{2}+X, \\
& Y^{2}=X^{5}-2 \sqrt{5} X^{4}+\frac{125}{18} X^{3}-2 \sqrt{5} X^{2}+X .
\end{aligned}
$$


Their common Igusa's invariants are

$$
\begin{aligned}
& i_{1}=\frac{32844064065625}{69984}=\frac{5^{5} \cdot 101^{5}}{2^{5} \cdot 3^{7}}, \\
& i_{2}=\frac{3219690625}{216} \quad=\frac{5^{5} \cdot 101^{3}}{2^{3} \cdot 3^{3}}, \\
& i_{3}=\frac{2806550125}{648} \quad=\frac{5^{3} \cdot 31 \cdot 71 \cdot 101^{2}}{2^{3} \cdot 3^{4}} .
\end{aligned}
$$

Example 3. $H=\left(\frac{3,5}{\mathbb{Q}}\right)=\langle 1, \mathrm{I}, \mathrm{J}, \mathrm{K}\rangle, D=15$.

We consider the PEL-type given by the following data:

$\Phi(\mathrm{I})=\left[\begin{array}{cc}\sqrt{3} & 0 \\ 0 & -\sqrt{3}\end{array}\right], \Phi(\mathrm{J})=\left[\begin{array}{ll}0 & 1 \\ 5 & 0\end{array}\right]$,

$\mu=-\mathrm{IJ}, \quad \delta=15$,

$\mathcal{O}_{15}=\mathbb{Z}\left[1, \mathrm{I}, \frac{1+\mathrm{J}}{2}, \frac{\mathrm{I}+\mathrm{IJ}}{2}\right]$

$\mathrm{V}=\left(\left(\frac{1}{4},-\frac{1}{4},-\frac{1}{4},-\frac{1}{4}\right),\left(\frac{1}{2}, \frac{1}{2}, 0,0\right)\right)$,

$\Phi\left(\mathcal{O}_{15}\right)=\mathbb{Z}\left[1_{2},\left[\begin{array}{cc}\sqrt{3} & 0 \\ 0 & -\sqrt{3}\end{array}\right],\left[\begin{array}{cc}-\frac{\sqrt{3}}{2} & -\frac{\sqrt{3}}{2} \\ \frac{5 \sqrt{3}}{2} & \frac{\sqrt{3}}{2}\end{array}\right],\left[\begin{array}{cc}-\frac{1}{2} & -\frac{1}{2} \\ -\frac{5}{2} & -\frac{1}{2}\end{array}\right]\right]$.

We have four SCM-points. Two of them have CM by $R(-15,1)$ :

$$
\tau_{1}=\frac{i(2 \sqrt{5}-\sqrt{15})}{5}, \quad \tau_{2}=\frac{15-5 \sqrt{3}+i(3 \sqrt{5}-\sqrt{15})}{30}
$$

and the other two have CM by $R(-15,2)$ :

$$
\tau_{3}=\frac{i \sqrt{5}}{5}, \quad \tau_{4}=\frac{5+2 i \sqrt{5}}{15}
$$

They define the points in the Siegel upper half-space

$$
\begin{array}{ll}
Z_{1}=\left[\begin{array}{cc}
i \sqrt{15} & \frac{-1+i \sqrt{15}}{2} \\
\frac{-1+i \sqrt{15}}{2} & \frac{i \sqrt{15}}{3}
\end{array}\right], & Z_{2}=\left[\begin{array}{cc}
\frac{-5+i \sqrt{15}}{4} & \frac{-21+i \sqrt{15}}{12} \\
\frac{-21+i \sqrt{15}}{12} & \frac{-5+i \sqrt{15}}{12}
\end{array}\right], \\
Z_{3}=\left[\begin{array}{ll}
\frac{i \sqrt{15}}{2} & -\frac{1}{2} \\
-\frac{1}{2} & \frac{i \sqrt{15}}{6}
\end{array}\right], & Z_{4}=\left[\begin{array}{cc}
\frac{i \sqrt{15}}{3} & -\frac{4}{3} \\
-\frac{4}{3} & \frac{i \sqrt{15}}{9}
\end{array}\right] .
\end{array}
$$


The points $\tau_{1}, \tau_{2}$ yield the genus 2 curves

$$
\begin{aligned}
Y^{2}=X^{5} & +\frac{7 \sqrt{5}}{4} X^{4}+\frac{35}{6} X^{3}+\frac{7 \sqrt{5}}{4} X^{2}+X \\
Y^{2}=X^{5} & -\frac{i \sqrt{5}}{4} \sqrt[4]{2111-168 i \sqrt{15}} X^{4}-\frac{3 \sqrt{15}}{2} X^{3} \\
& +\frac{i \sqrt{5}}{4} \sqrt[4]{2111+168 i \sqrt{15}} X^{2}+X
\end{aligned}
$$

Their common Igusa's invariants are

$$
\begin{aligned}
& i_{1}=-\frac{25000}{3}=-\frac{2^{3} \cdot 5^{5}}{3}, \\
& i_{2}=-\frac{9375}{2}=-\frac{3 \cdot 5^{5}}{2} \\
& i_{3}=-\frac{9875}{8}=-\frac{5^{3} \cdot 79}{2^{3}} .
\end{aligned}
$$

The points $\tau_{3}, \tau_{4}$ yield the genus 2 curves

$$
\begin{aligned}
Y^{2}=X^{5} & -\frac{1}{28} \sqrt{5(3439+240 \sqrt{15})} X^{4} \\
& +\frac{1}{294}(2140+97 \sqrt{15}) X^{3} \\
& -\frac{1}{28} \sqrt{5(3439+240 \sqrt{15})} X^{2}+X \\
Y^{2}=X^{5} & -\frac{\sqrt{5}}{28} \sqrt[4]{91861516+21983071 \sqrt{15}} X^{4} \\
& +\frac{11}{294} \sqrt{5(40772+9139 \sqrt{15})} X^{3} \\
& -\frac{\sqrt{5}}{4} \sqrt[4]{527116+135377 \sqrt{15}} X^{2}+X .
\end{aligned}
$$

Their common Igusa's invariants are

$$
\begin{aligned}
& i_{1}=\frac{230234596815794975000}{41523861603}=\frac{2^{3} \cdot 5^{5} \cdot 1559^{5}}{3 \cdot 7^{12}}, \\
& i_{2}=\frac{2877362908115625}{11529602}=\frac{3^{5} \cdot 5^{5} \cdot 1559^{3}}{2 \cdot 7^{8}}, \\
& i_{3}=\frac{3063998328865125}{46118408}=\frac{3 \cdot 5^{3} \cdot 1559^{2} \cdot 3361747}{2^{3} \cdot 7^{8}} .
\end{aligned}
$$

The quaternion algebras of discriminant $D=6,10,15$ are twisting quaternion algebras in the sense of Rotger. In these cases, forgetful mappings explain the coincidence of Igusa's invariants detected in the examples (cf. [Ro02], [Ro04]). 


\section{References}

[A104] M. Alsina, Binary quadratic forms and Eichler orders. Journées Arithmétiques Graz 2003 , in this volume.

[AlBa04] M. ALsina, P. BAYER, Quaternion orders, quadratic forms and Shimura curves. CRM Monograph Series 22. AMS, 2004.

[Ba02] P. BAYER, Uniformization of certain Shimura curves. In Differential Galois Theory, T. Crespo and Z. Hajto (eds.), Banach Center Publications 58 (2002), 13-26.

[Bu96] K. Buzzard, Integral models of certain Shimura curves. Duke Math. J. 87 (1996), 591-612.

[Gu02] J. GuÀrdiA, Jacobian nullwerte and algebraic equations. Journal of Algebra 253 (2002), 112-132.

[Gu] J. GUÀRDIA, Jacobian Nullwerte, periods and symmetric equations for hyperelliptic curves. In preparation.

[Ei55] M. EICHLER, Zur Zahlentheorie der Quaternionen-Algebren. J. reine angew. Math. 195 (1955), 127-151.

[HaMu95] K. Hashimoto, N. Murabayashi, Shimura curves as intersections of Humbert surfaces and defining equations of QM-curves of genus two. Tôhoku Math. J. 47 (1995), 271-296.

[Jo81] B.W. JoRdan, On the Diophantine Arithmetic of Shimura Curves. Thesis. Harvard University, 1981.

[Mi79] J.S. Milne, Points on Shimura varieties mod $p$. Proceed. of Symposia in Pure Mathematics 33, part 2 (1979), 165-184.

[Mo92] A. MORI, Explicit Period Matrices for Abelian Surfaces with Quaternionic Multiplications. Bollettino U. M. I. (7), 6-A (1992), 197-208.

[RV00] F. Rodríguez-VILLEgas, Explicit models of genus 2 curves with split CM. Algorithmic number theory (Leiden, 2000). Lecture Notes in Compt. Sci. 1838, 505-513. Springer, 2000.

[Ro02] V. ROTGER, Abelian varieties with quaternionic multiplication and their moduli. Thesis. Universitat de Barcelona, 2002.

[Ro03] V. ROTGER, Quaternions, polarizations and class numbers. J. reine angew. Math. 561 (2003), 177-197.

[Ro04] V. Rotger, Modular Shimura varieties and forgetful maps. Trans. Amer. Math. Soc. 356 (2004), 1535-1550.

[Sh67] G. ShImURA, Construction of class fields and zeta functions of algebraic curves. Annals of Math. 85 (1967), 58-159.

[Sh77] G. ShimurA, On the derivatives of theta functions and modular forms. Duke Math. J. 44 (1977), 365-387.

[Sh98] G. ShimURA, Abelian varieties with complex multiplication and modular functions. Princeton Series, 46. Princeton University Press, 1998.

[Vi80] M.-F. VignÉRAs, Arithmétique des algèbres de quaternions. LNM 800. Springer, 1980.

[We76] A. WeIL, Sur les périodes des intégrales abéliennes. Comm. on Pure and Applied Math. 29 (1976), 813-819.

Pilar BAYER

Facultat de Matemàtiques

Universitat de Barcelona

Gran Via de les Corts Catalanes 585. E-08007, Barcelona

E-mail : bayer@ub.edu

Jordi GuÀRDIA

Departament de Matemàtica Aplicada IV

Escola Politècnica Superior d'Enginyeria de Vilanova i la Geltrú

Avinguda Víctor Balaguer $\mathbf{s} / \mathbf{n}$

E-08800, Vilanova i la Geltrú

E-mail : guardia@mat.upc.es 